\title{
TERMINAL HINGE AXIS OF THE MANDIBLE - MODERN VIEWS
}

\author{
Nazarov Olimjon Jabborovich, Gaybullaeva Yulduz Hamidovna \\ olimnazarov@mail.ru \\ yulduz0706@gmail.com \\ ORCiD ID orcid.org/0000-0002-7144-7286 \\ ORCID ID orcid.org/0000-0003-4618-1559
}

Tashkent institute of postgraduate medical education, Tashkent, Republic of Uzbekistan

http://dx.doi.org/10.26739/2573-5616-2017-5-5-8

The article belongs to medicine, diagnostics in stomatology, orthodontics and includes face bow to transfer the position of a jaw model in interframe space of an articulator that is relative to its terminal ninge axis and a way of diagnostics at pathologies of tempo mandibular joints. To achieve the aimed goals clinical dental examination, dental computer tomography and a lateral cephalo analysis at volunteers is conducted.

Key words: Terminal ninge axis, face bow transfer, temporomandibular jont, dental articulator, diagnostics.

Normally, the movements of the lower jaw start from the position of the optimal maximum closure of the teeth. In this position, there should not be any load on the articular heads in the joint fossa. The articular heads should be adjacent to the intermediate zone of the articular discs, and the 
masticatory muscles should be in the myocentric position (that is, the position that ensures maximum efficiency with minimal energy expenditure). Ideally, with maximum closure of the teeth, the occlusal plane passes through the base point of the first cervical vertebra; and the forces resulting from the closing of the teeth in maximum contact are directed perpendicularly to the occlusal plane and parallel to the longitudinal axes of the chewing teeth on both sides.

It is considered that when the mouth is opened, the lower jaw first rotates around an imaginary stationary articulation axis passing through the joint heads, and then shifts downwards and forwards, and also laterally (when the jaw is shifted to the side).

Peter Dawson argues that "the axis of rotation passing through the medial poles of both joints causes the possibility of a pure rotation of the lower jaw around the stationary axis about the first $20 \mathrm{~mm}$ of the opening of the mouth" [5].

Jeffrey Okeson says, "The rotation of the lower jaw occurs when the mouth is opened and closed around the stationary rotation center. The rotation centers are located in the articular heads. In other words, the closing and opening of the teeth can occur without changing the position of the axes of the articular heads "[6].

According to the Kazai Guzai's Quadrant theorem [4], the axis of rotation of the lower jaw is located in the subarticular area between the first and second cervical vertebrae (when the teeth are closed in physiological occlusion). Gallo et al. analyzed the geometry of the movements of the lower jaw to determine the orientation and kinematics of the movement axis of rotation of the lower jaw in people with a normally functioning masticatory system when opening and closing the mouth. Researches have shown that "when opening and closing the mouth, the axis of rotation does not momentarily appear in the region of the articular heads and is often located even outside the lower jaw," and the main movement of the articular heads is translation, and not rotation [4].

Traditionally it is considered that when the mouth is opened, the jaw rotates around the hinge axis first. Quadrant theorem and Gallo's et al. research show that this is not quite so. Merlini and Palla in their studies 
have shown that with a clinically determined click in the joint, the opening movement begins with an expressed rotation. Expressed rotation is observed at the end of the closing movement. If the position of the lower jaw is incorrect, the articular heads, discs and muscles of the head, neck and face will have a pathological load [4].

Musculo-articular dysfunction causes patients unpleasant sensations in the maxillofacial area and facial pain. The causes of these complaints in many cases are anomalies of occlusion. The basis for the diagnosis of such disorders is a functional analysis of the dentofacial system, whose main goal is to establish a connection between articulatory disorders of the lower jaw and the pathology of occlusion, masticatory muscles and temporomandibular joint (herein after TMJ).

In V.A. Khvatova's opinion, musculo-articular dysfunctions with occlusal disorders can be represented as follows. Due to the altered muscular function, the movements of the lower jaw are carried out so as to avoid occlusal obstructions. There is an asymmetry of muscular activity and topography of the head of the TMJ, trauma of the nerve endings of the joint capsule, the disc zone, a violation of the hemodynamics of the TMJ [2].

Thus, special importance for the prevention and diagnosis of muscular-articular dysfunctions is given to the definition of the topography of the TMJ heads, in particular to the location of the axial axis of the mandible of the patient.

To eliminate errors in the coordinates of the axis of the head of the lower jaw, a face bow is traditionally used. Failure to apply or position to the wrong point of the articulated axis of the face bow arc can lead to errors in the occlusion and will be the more, the more errors in the orientation of the models in the articulator are allowed. Although errors in face bow transfer can be partially compensated for by an accurately made occlusal register or a decrease in the inclination and height of the hillocks on the restoration produced. But, nevertheless, if in the process of prosthetics the mounds are modeled more than 20 degrees (and they should not be less) and if the entire dentition is restored, then the use of the facial arch is necessary.

However, in these known methods, the position of the point of the hinge axis of the head of the lower jaw is placed on the basis of probabilistic 
presumptive data or on the basis of palpation, which is not always applicable to a particular patient and corresponds to the place of the actual dermal projection point of this axis.

The purpose of our research was to determine the true point of the axis of rotation of the head of the mandible (terminal hinge axis).

To achieve this goal, we conducted a clinical and instrumental examination of a group of volunteers (35 people, 20 men and 15 women aged 30 to 40 years.). When choosing volunteers, attention was drawn to the lack of clinical signs of functional disorders in the TMJ, which consisted in the following: the symmetrical face configuration; palpation of the joint at rest, during opening, closing is painless; the opening of the mouth is free, the degree of opening of the mouth was $45-50 \mathrm{~mm}$ (average $47.6 \mathrm{~mm}$ ); movements of the lower jaw from the position of the central occlusion - to the lateral, to the anterior and posterior directions with closed dentition is smooth; sound phenomena, on the part of the joint, are absent; movements of the lower jaw when opening and closing the mouth are strictly vertical, without deviation to the sides and zigzag shifts; palpation of the masticatory muscles is painless.

Examination of the maxillofacial system began with examination of the skin, while paying attention to the swelling and flushing of the skin in the area of the TMJ, the presence of cicatricial changes. This allows us to judge the transferred operations and injuries, as well as the presence of inflammation phenomena in this area. The height of the lower part of the face was determined in the position of the central occlusion and compared with the height of the physiological rest of the lower jaw. Particular attention was paid to the height of the lower part of the face in the presence of various designs of dentures in patients, as they create new occlusal relationships in the oral cavity and change the spatial position of the lower jaw. Determined the trajectory of the movement of the lower jaw, its smoothness and symmetry. The degree of opening of the mouth was established, for this purpose, a ruler was used.

Palpation of the TMJ was carried out through the skin and from the external auditory canal. Palpated masticatory muscles, neck muscles and 
waist of the upper limb. The presence of pain during palpation was an indication of the presence of trigger points (TP).

An important part of the functional analysis of teeth, jaws and MJ is radiography in particular lateral teleradiography and dental threedimensional computed tomography. Dental computed tomography of the maxillofacial area qualitatively improves the diagnostic process of pathologies of temporomandibular joints associated with functional disorders.

The three-dimensional image is stored in the memory of a computer tomograph and allows the dentist or the radiologist to obtain any section of the zone of interest and any projection.

Based on the data obtained from the dental computed tomography of the temporomandibular joints, J. Carlson's hypothesis was confirmed which, based on the Kazey Guzai Quadrant Theorem, the results of Gallo's study and his own numerous clinical studies, asserts that the axis of rotation of the lower jaw is not located in the joints.

All 35 surveyed analyzed the topography of the TMJ to determine the orientation and kinematics of the displacement of the axis of rotation of the lower jaw when the mouth was opened with an interocclusion distance of $7-10 \mathrm{~mm}$ in the region of the anterior teeth and closed dentition in the usual occlusion.

CT data showed that the axis of rotation of the lower jaw is a point located in the lower part of the billamarine zone behind the articular heads. This means that when the lower jaw is opened to a distance of 5-7 mm, it rotates around the above point.
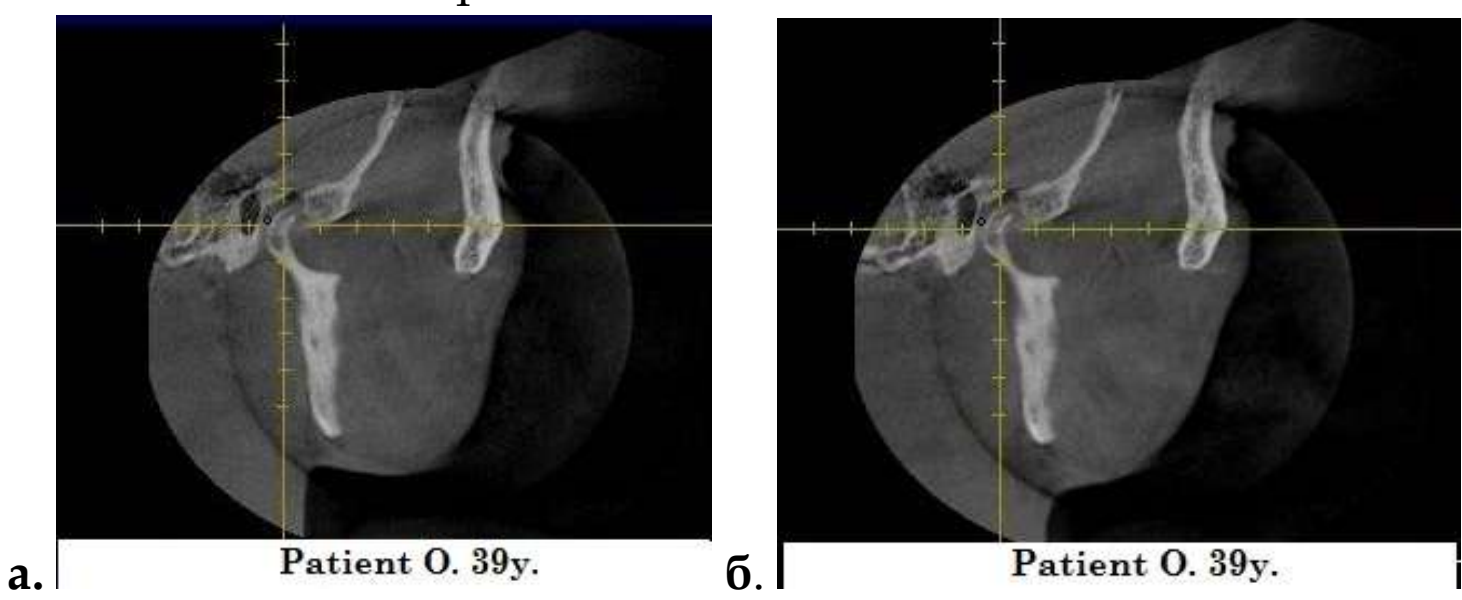

Fig.1 Point of the articular axis of the head 
of the lower jaw at CO (a) and CR on 6mm (b).

The transfer of the position of the upper and lower jaws of dental patients to the interframe space of the articulator for the diagnosis and treatment of prosthetic and orthodontic patients is focused mainly on the projection point of the hinge axis of the mandible. When determining the projection point of the position of the hinge axis on the skin of the face during an average anatomical transfer, usually firstly found the approximate center of rotation of the condyle. It is generally accepted that it is on the line (Fisher) connecting the outer corner of the eye with the top of the tragus of the ear approximately $13 \mathrm{~mm}$ ahead of the external auditory canal, which in the lateral x-ray corresponds to the center of the articular head of the mandible. Joint articulations of the face bow of orientaed to this projection of the point. In this case, the error of the axis of rotation of the condyle of the lower jaw is 3 to $6 \mathrm{~mm}$.

Based on the above, we recommend using a more accurate way to determine the projection of the point of the hinge axis on the skin, proposed by M. Nasyrov (2005). The procedure consists of the following sequence:

1. On the patient under examination, along the line from Tragus medialis to the deepest point of the orbit, put the first point $(5 \mathrm{~mm}$ anterior to Tragus medialis along the depicted line) on the patient's skin with radiopaque substance or glue an aluminum circle or a steel ball.

2. The second point is placed $2 \mathrm{~mm}$ above the first point on the line originating from the first point, which is perpendicular to the line passing from Tragus medialis to the deepest point of the orbit.

3. The third and fourth dermal points are located on the sides of the second point at a distance of $2 \mathrm{~mm}$ on a line parallel to the line running from Tragus medialis to the deepest point of the orbit.

4. On the lateral cephalo, draw a line from the point Po (the Porion) to the point $\mathrm{O}$ (the lowest edge of the orbit). From this line, draw a perpendicular line down, passing through the points Ar (the point of intersection of the shadow of the arch zygomaticus bone with the posterior edge of the frame of the lower jaw).

5. Determined a point $2 \mathrm{~mm}$ above the point $\mathrm{Ar}$, which is the axis of rotation of the head of the patient's lower jaw, and its coordinates relative to 
the projections of dermal points on this radiograph are transferred to the patient's skin in the area of previously deposited dermal points (Fig. 2).

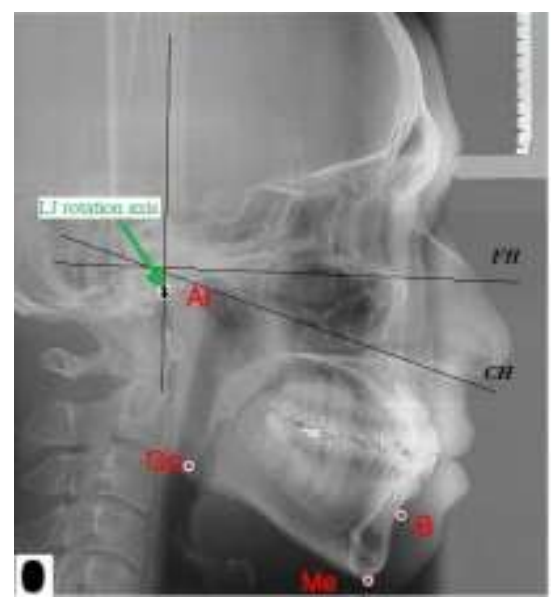

Fig.2. Projection of the point of the hinged axis of the lower jaw on the lateral cephalogram.

This method allows to maintain accuracy when transferring the position of the upper jaw of the patient to the articulator, thereby significantly reducing the errors in the diagnosis and treatment of dental patients.

Someone will react critically to new principles, but criticism will promote the dissemination and improvement of these principles. Someone will start to apply new concepts in their practice, and we very much welcome such a decision - the decision to throw off the shackles of old traditions and to think more broadly, to adopt and to evaluate innovations in dentistry. 


\section{References}

1. Nasyrov M.M. Face bow. "Clinical stomatology", 2005/4/36.

2. Khvatova V.A. Clinical gnathology. - Moscow: Medicine, 2005.-296 p.

3. Khvatova V.A., Khvatov I.L. The importance of graphical research methods in diagnosis of temporomandibular joint dysfunction. // Maestro of stomatology. - 2002.- N2. -C.12-15.

4. Carlson J. E., Physiologic occlusion, Midwest Press, 2009.

5. Dawson, P, Commentary from the Dawson Center for Advanced Dental Study, June, 2000 St. Petersburg, FL 33701.

6. Okeson, Jeffery P., Management of Temporomandibular Disorders and Occlusion, 4th Edition, (C. V. Mosby Co., 1998.). 